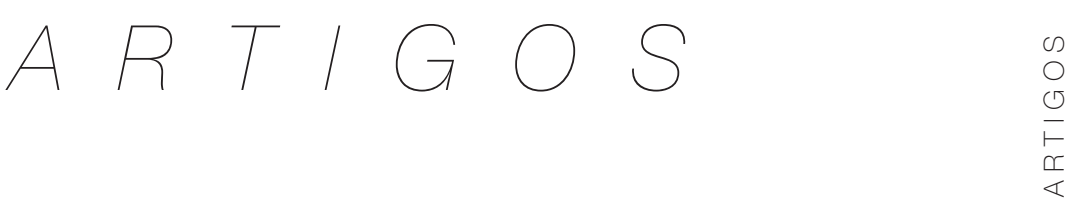

\title{
ESPERANÇA EM IDOSOS: UMA EXPLICAÇÃO BASEADA NOS VALORES HUMANOS
}

Patricia Nunes Fonseca ${ }^{1}$

Gildevan Estrela Dantas²

Gabriel Lins de Holanda Coelho ${ }^{3}$

Thayro Andrade Carvalho ${ }^{4}$

Alisson de Meneses Pontes ${ }^{5}$

resumo

Este estudo teve como objetivo conhecer como a esperança se relaciona com os valores humanos em idosos. A esperança é fundamental no processo de envelhecimento, possibilitando uma perspectiva positiva quanto ao futuro. Para sua melhor compreensão, faz-se

\footnotetext{
1 Graduada em Psicologia. Doutora em Psicologia Social. Professora da Universidade Federal da Paraíba (UFPB) vinculada ao Departamento de Psicopedagogia e à Pós-Graduação de Psicologia Social. E-mail: pnfonseca.ufpb@gmail.com

2 Graduado em Psicologia. Mestre em Psicologia Social. Professor das Faculdades Integradas de Patos (FIP) vinculado ao curso de Psicologia. E-mail: gildevanteo@hotmail.com

3 Graduado em Psicologia. Mestre em Psicologia Social. Doutorando da Cardiff University. E-mail: gabriel_lins@hotmail.com

4 Graduado em Psicologia. Mestrando em Psicologia Social da Universidade Federal da Paraíba (UFPB). E-mail: thayrocarvalho@gmail.com

5 Graduado em Psicologia. Mestre em Psicologia Social pela Universidade Federal da Paraíba (UFPB). Professor das Faculdades Integradas de Patos (FIP) vinculado ao curso de Psicologia. E-mail: alisson.mpontes@hotmail.com
} 
necessário considerar os valores humanos que atuam orientando e guiando o comportamento humano, estando sempre presentes na sociedade e sofrendo alterações à medida em que indivíduo vai amadurecendo. Participaram 218 idosos, com idade média de 68 (DP $=6,65)$ e a maioria do sexo feminino $(61,9 \%)$. Estes responderam ao Questionário dos Valores Básicos, à escala de esperança de Herth e a perguntas demográficas. Realizaram-se correlações (r de Pearson) entre as subfunções valorativas e o escore total de esperança, além de uma regressão múltipla. Observaram-se correlações significativas em todas as subfunções: interativa $(r=0,43 ; p<0,01)$, existência $(r=$ 0,39; $p<0,01)$, suprapessoal ( $r=0,38 ; p<0,01)$, normativa $(r=0,31$; $p<0,01)$; realização $(r=0,20 ; p<0,01)$ e experimentação $(r=0,19$; $p<0,01)$, respectivamente. Na regressão, as dimensões interativa, normativa, existência e suprapessoal foram preditores significativos do escore total de esperança, tendo variância explicada de 26\% $[F(4 ; 206)=19,39 ; p<0,001]$. Os resultados sugerem a importância dos valores humanos para a promoção de uma vida mais saudável e com maior esperança na terceira idade, principalmente quando refere-se a valores centrais e sociais. Conclui-se que os objetivos tenham sido alcançados, contribuindo com a literatura sobre a qualidade de vida na terceira idade.

palavras-chave

Esperança. Valores Humanos, Idosos.

1 Introdução

O envelhecimento da população é um fenômeno social caracterizado pelo aumento da proporção de idosos em relação à população total (IBGE, 2012). Nos países em desenvolvimento como o Brasil, o crescimento ocorre de forma acelerada, exigindo, do governo, políticas voltadas à pessoa idosa. Segundo a Organização das Nações Unidas (ONU, 2013), este processo de transição demográfica é único e irreversível, estimando-se que a proporção de pessoas com 60 anos ou mais deve duplicar até 2050, chegando a uma população bastante expressiva de idosos: cerca de dois bilhões. Corroborando estas estimativas, o IBGE (2012) mostra que, em um intervalo de dez anos, o número de idosos no Brasil passou de 15,5 milhões, em 2001, para 23,5 milhões, em 2011. 
Este expressivo avanço da população idosa tem sido associado à combinação de uma série de fatores, dentre os quais se destacam: a redução dos índices de mortalidade, as melhorias das condições de alimentação, os avanços na medicina, o aumento da renda, o controle de vetores causadores de doenças infecciosas e a ampliação dos serviços de saúde para a pessoa idosa. Ademais, ainda pode-se contabilizar a redução das taxas de fecundidade relacionada às mudanças nos padrões familiares, estes advindos do acesso a meios contraceptivos e da maior participação feminina no mercado de trabalho (BLOOM, 2011).

No Brasil, a partir da Constituição Federal de 1988, foram desenvolvidas políticas públicas específicas para a pessoa idosa, a exemplo do Estatuto do Idoso (BRASIL, 2003), que vieram estabelecer os direitos das pessoas com idade igual ou superior aos 60 anos, determinar obrigações das entidades assistenciais e estabelecer penalidades para situações de desrespeito aos idosos.

Em 2006, por influência das decisões tomadas na V Conferência Internacional sobre a Promoção da Saúde realizada no México, os gestores do SUS assumiram o compromisso do Pacto pela Saúde, tendo como componente o Pacto pela Vida. Este programa tem como prioridade a saúde do idoso e a promoção do envelhecimento ativo e saudável com serviços de atenção domiciliar e a implantação da Política Nacional de Saúde da Pessoa Idosa (PNSPI) (BRASIL, 2006). Esta visa recuperar, manter e promover a autonomia e a independência dos idosos (IESS, 2013).

No entanto, apesar destas políticas colaborarem para o aumento da expectativa de vida dos idosos, é perceptível a dificuldade de operacionalização dos serviços em razão da insuficiência de residências ou centros de recreação para atender à demanda de idosos, ficando restrita a uma minoria da população (KÜCHEMANN, 2012).

Embora haja um aumento no número de idosos e, também, na produção de conhecimentos acerca deste contingente populacional, o envelhecimento humano ainda é visto como uma história de perdas físicas, mentais e sociais, o que, geralmente, impõe limitações ao indivíduo. Por conseguinte, traz a necessidade de compreender a relação do idoso com os mecanismos de ajustamento e adaptação a essa nova fase do desenvolvimento humano (SOMMERHALDER, 2010). Nessa etapa, é importante para o idoso reconhecer estas limitações que surgem com o avanço da idade, mas também entender que os desafios precisam ser enfrentados. Para que isto ocorra, há necessidade de procurar aumentar o nível de esperança, visando envelhecer com um melhor bem-estar subjetivo (SNYDER; RAND; SIGMON, 2002). 
A esperança é um conceito multidimensional e dinâmico, o qual impulsiona o indivíduo a agir, se mover e alcançar seus objetivos. De acordo com Snyder, Rand e Sigmon (2002), ela tem sido estudada como uma variável que se relaciona com a percepção em frente a metas/objetivos. Estes autores definem a esperança como um traço cognitivo que engloba crenças positivas quanto à realização de objetivos pessoais, algo como um sentimento de sucesso quanto ao futuro. Estes objetivos pessoais podem variar, surgindo de diversas maneiras, como o desejo de ir a algum lugar, experimentar ou criar algo.

Frankl (2007) afirma que há conexão existente entre a esperança e a busca de sentido. Nos campos de concentração, enquanto os prisioneiros perseguiam um objetivo, ou seja, uma esperança que iluminasse sua vida, eles podiam sobreviver. A esperança é considerada fundamentalmente como um processo de escolha de sentido pessoal na vida.

Segundo Geiger e Kwon (2010), a esperança serve como um fator de proteção a eventos estressantes pelo qual indivíduos que apresentam esperança elevada demonstram um melhor ajustamento psicológico, baixos sintomas depressivos e maior habilidade para resolver problemas. Ademais, demonstram maior nível de perseverança para alcançar suas metas e experimentar emoções positivas, enquanto aqueles que exibem nível baixo de esperança tendem a ver as situações de forma negativa, o que pode leva-los a vivenciar tristeza e depressão.

$\mathrm{Na}$ atualidade, é possível encontrar trabalhos em que a esperança é percebida como um forte recurso psicológico para as pessoas lidarem com questões negativas como, por exemplo, estudos com pacientes portadores de câncer (KAVRADIM; OZER; BOZCUK, 2013). Outro bom exemplo da funcionalidade da esperança são pesquisas que associam este fator à recuperação de pessoas com doença mental grave (VAN GESTEL-TIMMERMANS et al., 2010) ou, até mesmo, com obesidade (NOTHWEHR; CLARK; PERKINS, 2013).

Em estudo realizado por Mestre (2011) com pacientes idosos que apresentavam problemas renais crônicos, foi verificado que manter a esperança resulta em melhorias nos níveis de qualidade de vida dos doentes. Ademais, em outra pesquisa com pacientes idosos semelhantes ao estudo anterior, foi possível verificar que manter a esperança diante do processo de envelhecimento é um recurso para aquisição de resiliência (ORLANDI et al., 2012). Com isso, evidencia-se o efeito benéfico que a esperança apresenta para a saúde das pessoas, já que auxilia na capacitação do indivíduo para suportar as situações de crise e na determinação de objetivos futuros.

Ter esperança é reconhecer as limitações nas situações, acreditando ao mesmo tempo em que as oportunidades existem a fim de superar as 
adversidades da vida. É importante, ainda, destacar que, com o passar dos anos, os objetivos de vida tendem a ser alterados na medida em que mudam as suas prioridades e valores, que regem as ações e a percepção de mundo. Entre os 45 e 50 anos, há uma mudança considerável nesta estrutura, em que há uma reavaliação dos valores em termos de crescimento pessoal, aquisição de uma maior autenticidade e de uma visão mais realista sobre si próprio, os quais são consolidados na velhice (DEVESA, 2005). Desta forma, para uma melhor compreensão da esperança em idosos, faz-se necessário considerar quais os valores humanos que orientam e guiam os seus comportamentos.

Os valores humanos podem ser entendidos como aspectos psicológicos que cumprem as funções de orientar o comportamento humano e representar cognitivamente as suas necessidades, transcendendo situações específicas e sendo desejáveis e relativamente estáveis (GOUVEIA et al., 2011). Eles influenciam as ações e as atitudes humanas, servindo como padrão avaliativo do comportamento (GOUVEIA, 2003). Optou-se por utilizar a Teoria Funcionalista dos Valores Humanos nesta pesquisa, por ser considerada integradora e mais parcimoniosa, além de teoricamente mais fundamentada do que outros modelos teóricos, possuindo estudos realizados nos mais diversos contextos (e.g., salas de aula e centros religiosos, políticos e policiais) e em mais de 20 países (e.g., Alemanha, Argentina, Brasil, Espanha, Israel, México e Reino Unido) (GOUVEIA, 2013).

Gouveia (2003) identificou duas funções consensuais dos valores: (1) guiar as ações humanas e (2) expressar as suas necessidades. A primeira função é conceituada como tipo de orientação, que é subdividida em social, central e pessoal. Indivíduos que tendem a apresentar uma orientação mais pessoal são mais centrados neles mesmos, egocêntricos e com foco intrapessoal. Os indivíduos que apresentam orientação mais social são mais centrados na sociedade, enfatizam o grupo e têm um foco interpessoal (GOUVEIA, 2003). Alguns valores são compatíveis tanto com os valores pessoais como com os sociais, sendo denominados centrais, funcionando como espinha dorsal dos outros valores e evidenciando a polarização entre as necessidades mais básicas como comer e beber, e mais elevadas como cognitivas e estéticas (GOUVEIA, 2013).

A segunda função definida por Gouveia (2003) é identificada pela dimensão funcional, expressando o tipo de motivador que representa as necessidades humanas, podendo ser materialista (pragmático) ou humanitário (idealista). A ênfase em valores materialistas indica orientação para metas específicas e regras normativas, enquanto os valores humanitários indicam uma orientação mais universal baseada em ideias e princípios mais abstratos (GOUVEIA, 2013). O cruzamento destas duas funções dos valores humanos, em um modelo $3 \times 2$, 
pode ser observado na Figura 1, que permite identificar as seis subfunções valorativas descritas a seguir (GOUVEIA et al., 2011):

1) Existência: tipo de motivador materialista e com a orientação central. Representa as necessidades básicas do ser humano como as fisiológicas (comer e beber) e de segurança. O propósito principal é assegurar as condições necessárias para sobrevivência biológica e psicológica dos indivíduos. Esta subfunção é a mais importante do motivador materialista, sendo fonte de outras duas subfunções que também representam este motivador (realização e normativa). Os três valores indicadores desta subfunção são estabilidade pessoal, saúde e sobrevivência.

2) Realização: subfunção encontrada no cruzamento do tipo de motivador materialista com a orientação pessoal. Representa pessoas imediatistas, que focam em realizações próprias e materiais, na praticidade em decisões e comportamentos. Estes valores são mais tipicamente encontrados por jovens adultos, em sua fase de produtividade, ou indivíduos em contextos mais disciplinares e formais (ROKEACH, 1973). Os valores indicadores desta subfunção são êxito, poder e prestígio.

3) Normativa: cruzamento do tipo de motivador materialista com a orientação social. Representa a importância de preservar a cultura e as normas sociais. A necessidade de controle e as pré-condições para alcançar as necessidades (MASLOW, 1954) são cognitivamente representadas por valores desta subfunção. Os indicadores desta subfunção são obediência, religiosidade e tradição.

4) Suprapessoal: encontrada no cruzamento do tipo de motivador idealista com a orientação central. Representa as necessidades estéticas e de cognição, assim como a necessidade de autorrealização (MASLOW, 1954). A pessoa que endossa uma orientação central e um motivador idealista costuma pensar de forma mais geral e ampla, tomando decisões e se comportando a partir de critérios universais (SCHWARTZ, 1992). Beleza, conhecimento e maturidade são os três valores indicadores desta subfunção.

5) Experimentação: subfunção encontrada no cruzamento do tipo de motivador idealista com a orientação pessoal. Representa a necessidade de excitação ou princípio do prazer no sentido amplo. Tais valores contribuem para a promoção de mudança e inovação na estrutura das organizações sociais, sendo mais endossados por jovens. Os valores desta subfunção são emoção, prazer e sexualidade.

6) Interativa: motivador idealista com a orientação social. Representa as necessidades de sociabilidade, amor e afiliação. As pessoas guiadas por esta subfunção dão maior importância aos demais, enfatizando estabelecer e manter 
as relações entre as pessoas e realçando características mais afetivas e abstratas. Os valores indicadores desta subfunção são afetividade, apoio social e convivência.

Figura 1 - Facetas, dimensões e subfunções dos valores básicos.

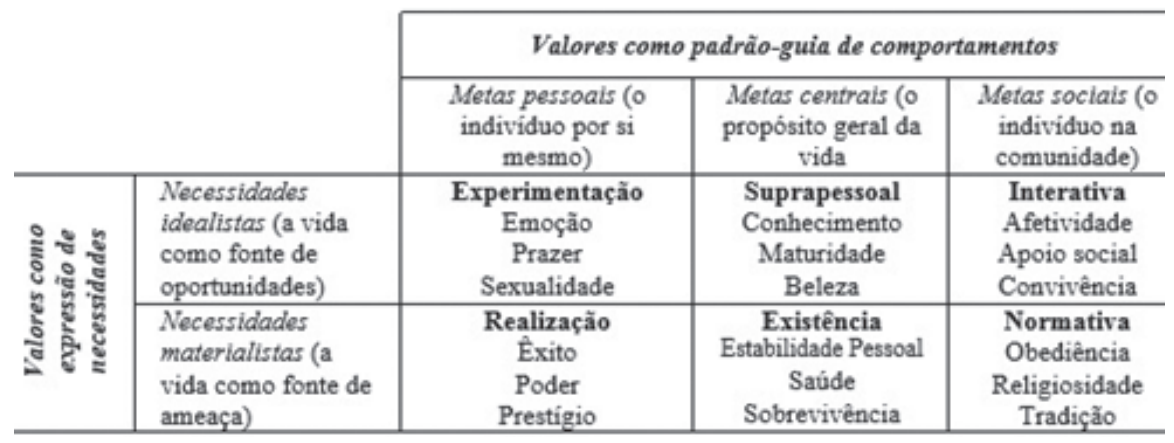

Fonte: Gouveia (2013)

Cheavens e Gum (2000) apontam várias adversidades comumente enfrentadas no processo de envelhecimento como declínio físico, psicológico, perda de parentes, amigos, entre outras. Apesar destas adversidades, observou-se, nos últimos anos, um aumento na expectativa e qualidade de vida da população mundial, o que também resultou em um crescimento da faixa etária idosa. Este crescimento, segundo os autores, evidencia a importância e necessidade do estudo da esperança na terceira idade uma vez que é associada a fatores como qualidade de e satisfação com a vida e envelhecimento saudável. Sabendo desta importância da esperança no processo de envelhecimento e da relevância dos valores humanos enquanto construto fundamental no comportamento e na motivação das pessoas, objetivou-se compreender a relação entre os construtos e a maneira em que os valores humanos podem influenciar na percepção do indivíduo perante as dificuldades sobrepostas pelo envelhecimento.

\section{Método \\ 2.1 Participantes}

Contou-se com a participação de 218 idosos da cidade de João Pessoa, Paraíba. A média de idade foi de 68 anos ( $\mathrm{DP}=6,65)$, sendo a maioria do sexo feminino $(61,9 \%)$, casado $(44 \%)$, com filhos $(89 \%)$, aposentado $(78,8 \%)$ e católico $(65,9 \%)$. Esta foi uma amostra de conveniência (não probabilística), 
tendo participado pessoas que se enquadravam nos seguintes critérios: ter idade igual ou acima de 60 anos, ser alfabetizado, ter condições de ler e compreender os itens dos instrumentos e não apresentar problemas de visão que impedissem a leitura.

\subsection{Instrumentos}

Foram utilizados a escala de esperança de Herth e o Questionário de Valores Básico, detalhadas a seguir, além de um questionário sociodemográfico.

A escala de esperança de Herth (EEH) trata-se de um instrumento de origem estadunidense criado por Herth (1992) e validado para a língua portuguesa por Sartore e Grossi (2008). É composto por 12 itens (e.g., Eu estou otimista quanto à vida, Eu tenho planos a curto e longo prazos e Eu sei onde quero ir) respondidos em uma escala Likert de quatro pontos, variando de 1 (Discordo Completamente) a 4 (Concordo Completamente). O escore total da escala pode variar de 12 a 48 pontos, sendo que quanto maior o escore, mais alto é o nível de esperança. A escala apresentou um alfa de Cronbach de 0,85. Este instrumento foi escolhido por abordar a temática em estudo, apresentar propriedades psicométricas satisfatórias e já ser validado na população brasileira.

O Questionário dos Valores Básicos (QVB) é um questionário criado e validado por Gouveia $(2003,2013)$ que contém itens que avaliam o grau de importância que cada valor tem como um princípio-guia para a vida da pessoa. É composto por 18 itens, dentre os quais servem como exemplos "Conhecimento" (Procurar notícias atualizadas sobre assuntos pouco conhecidos; tentar descobrir coisas novas sobre o mundo) e "Êxito" (Obter o que se propõe; ser eficiente em tudo que faz), agrupados equitativamente em seis subfunções valorativas, que são Experimentação, Suprapessoal, Interativa, Realização, Existência e Normativa; e respondidos em uma escala de 7 (sete) pontos, variando de 1 (Nenhuma importância) a 7 (Extremamente importante). A escala apresenta evidências de validade fatorial e consistência interna acima de 0,60 (MEDEIROS, 2011). Este instrumento foi adotado para este estudo por ser construído a partir da Teoria Funcionalista dos Valores Humanos, adotada neste estudo e relatada anteriormente, e por apresentar bons índices psicométricos e ser validado para o Brasil.

O questionário sociodemográfico apresenta perguntas sobre o sexo, a idade, a escolaridade, o estado civil, a religião e se possuem filhos, que permitiram caracterizar melhor a amostra. 


\subsection{Procedimentos}

Os pesquisadores contataram os responsáveis pelos centros de recreação e grupos de melhor idade a fim de solicitar autorização para convidar os idosos a participarem do estudo. Após a exposição dos objetivos do estudo para os idosos, foi comunicado que a participação na pesquisa seria voluntária e não haveria nenhum prejuízo caso o indivíduo resolvesse abandonar o estudo a qualquer momento. Também foi informado o caráter anônimo e confidencial das respostas, conforme o disposto na Resolução 466/12 do Conselho Nacional de Saúde. Os idosos que concordaram em participar foram solicitados a ler e preencher um Termo de Consentimento Livre e Esclarecido e, em seguida, a responder um questionário autoaplicável, no qual eram fornecidas instruções gerais sobre sua utilização. Não obstante, os responsáveis pela coleta permaneceram ao lado dos idosos a fim de esclarecer qualquer dúvida sobre a forma de responder. Ao término da aplicação, foram dirigidos os agradecimentos aos participantes. Em média, foram necessários 20 minutos para completar sua participação.

\subsection{Análise de dados}

Foi utilizada a versão 22 do software estatístico SPSS para a tabulação e a análise dos dados. Além de estatísticas descritivas, foram efetuadas correlações entre as dimensões valorativas e o escore total de esperança e uma regressão linear múltipla do tipo stepwise, utilizando as subfunções dos valores humanos como variáveis antecedentes e o escore total de esperança como variável consequente.

\section{Resultados}

Primeiro, realizou-se uma análise descritiva para observar a média do escore total da escala de esperança de Herth. Podendo variar entre 12 e 48 pontos, a média observada foi de 38,12 (DP =4,52). Foi realizado um teste $t$ de student para observar se existia diferença quanto ao sexo dos participantes, mas os resultados não foram estatisticamente significativos. Logo após, foi realizada uma análise descritiva a fim de observar os valores das dimensões do QVB, que podem variar de 1 a 7 , sendo apresentados na Tabela 1: 
Tabela 1 - Médias das dimensões dos Valores Humanos.

\begin{tabular}{l|c|c}
\hline & Média (M) & Desvio-padrão (DP) \\
\hline Interativa & 5,75 & 0,71 \\
\hline Experimentação & 4,35 & 1,12 \\
\hline Realização & 4,73 & 0,98 \\
\hline Existência & 6,04 & 0,64 \\
\hline Suprapessoal & 5,10 & 0,89 \\
\hline Normativa & 5,89 & 0,69
\end{tabular}

Fonte: Dados da pesquisa.

Após as análises iniciais, foi realizada uma correlação $r$ de Pearson para verificar uma possível correlação entre as seis dimensões dos valores humanos e o escore total da escala de esperança de Herth. Observou-se que a subfunção Interativa foi a que apresentou maior correlação com a escala de esperança de Herth $(r=0,43 ; p<0,01)$, sendo seguida pelas subfunções existência $(r=0,39 ; p$ $<0,01)$, suprapessoal $(r=0,38 ; p<0,01)$, normativa $(r=0,31 ; p<0,01)$, realização $(r=$ $0,20 ; p<0,01)$ e experimentação $(r=0,19 ; p<0,01)$.

Por fim, realizou-se uma regressão múltipla do tipo stepwise com as seis dimensões abordadas na Teoria Funcionalista dos Valores Humanos como preditores do escore total da escala de esperança de Herth. O melhor modelo apresentado abarcou as dimensões interativa, normativa, existência e suprapessoal, tendo variância explicada de $26 \%[F(4 ; 206)=19,39 ; p<0,001]$. Os resultados podem ser observados na Tabela 2:

Tabela 2 - Regressão múltipla para o escore total de esperança.

\begin{tabular}{l|c|c|l|c|c}
\hline Valores humanos & $\mathbf{R}$ & $\mathbf{r}^{2}$ Ajustado & $\mathbf{F}$ & Betaz & $\mathbf{t}$ \\
\hline Interativa & 0,43 & 0,18 & $\mathrm{~F}(1 ; 209)=47,43^{* \star *}$ & 0,43 & 6,89 \\
\hline Normativa & 0,48 & 0,22 & $\mathrm{~F}(2 ; 208)=31,24^{* \star *}$ & 0,22 & 3,53 \\
\hline Existência & 0,50 & 0,24 & $\mathrm{~F}(3 ; 207)=23,58^{* \star *}$ & 0,18 & 2,57 \\
\hline Suprapessoal & 0,52 & 0,26 & $\mathrm{~F}(4 ; 206)=19,39^{\star * \star}$ & 0,16 & 2,1 \\
\hline
\end{tabular}

Nota: ${ }^{\star \star \star} \mathrm{p}<0,001$

Fonte: dados da pesquisa.

\section{Discussão}

O objetivo do presente estudo foi observar as relações existentes entre as seis dimensões apresentadas na Teoria Funcionalista dos Valores Humanos 
(GOUVEIA, 2003) e o escore total da escala de esperança de Herth (EEH). Para tanto, teve-se como ponto de partida que os valores estão presentes em todos os estágios de desenvolvimento e que, em cada estágio, alguns valores se apresentam mais importantes para alguns indivíduos do que para outros. Além disso, na medida em que o indivíduo amadurece, tanto biológica como psicologicamente, estas prioridades seguem em constante processo de mudança (VIONE, 2012).

No que se refere às subfunções valorativas, verificou-se que o escore total da escala de esperança de Herth apresentou correlações estatisticamente significativas com todas as dimensões dos valores humanos (experimentação, realização, existência, suprapessoal, normativa e interativa). Estes resultados demonstram a importância dos valores humanos na terceira idade, atuando de maneira estreita com a esperança e ajudando para a promoção de uma melhor qualidade de vida.

A dimensão interativa, caracterizada pelas necessidades de sociabilidade, amor e afiliação, na ênfase em estabelecer relações com outras pessoas (GOUVEIA, 2013), foi a que apresentou maior correlação com o escore total de esperança. Esta dimensão abarca os valores de afetividade, apoio social e convivência (GOUVEIA, 2003), evidenciando a necessidade de sentir-se pertencente a algum grupo no qual se possa conviver diariamente e obter ajuda, além de compartilhar com outras pessoas os seus êxitos e fracassos. Segundo Stirling (2010), o aumento da rede social colabora para a qualidade de vida do idoso na medida em que permite a este sentir-se parte da sociedade e compartilhar suas esperanças com seu grupo de pertença.

A segunda dimensão que apresentou maior correlação com os níveis de esperança foi a existência, composta pelos itens estabilidade, saúde e sobrevivência, representando as necessidades mais básicas (GOUVEIA et al., 2011). Este resultado mostra que a esperança está diretamente correlacionada com o bem-estar físico e psicológico do idoso, assegurando sua sobrevivência biológica e psicológica. Quanto mais os valores dos idosos forem guiados por princípios que buscam uma melhora física e mental, maiores serão seus níveis de esperança. Stirling (2010) também ressalta a importância da sobrevivência biológica e psicológica do sujeito uma vez que para um envelhecimento ocorrer de forma saudável, é essencial que o idoso apresente bases seguras que lhe permitam continuar com o padrão de vida outrora apresentado.

No que tange à subfunção suprapessoal, esta representa as necessidades ligadas a padrões estéticos, cognição e autorrealização. Estes valores descrevem uma satisfação consigo mesmo, na qual os indivíduos tendem a perceber uma utilidade em suas vidas, tendo sentido existencial (GOUVEIA et al., 2011). Pessoas que apresentam estes valores como guias tendem a tomar decisões 
e utilizar critérios universais, caracterizando, na maioria das vezes, pessoas mais velhas (VIONE, 2012). O resultado sugere que uma percepção positiva do idoso quanto a si próprio fornece boas perspectivas quanto ao seu futuro.

A subfunção normativa é composta pelos itens obediência, religiosidade e tradição, representando o desejo de controle e as precondições para alcançar as necessidades básicas humanas (MEDEIROS, 2011). Este resultado pode ser explicado pelo fato de o idoso estar no último estágio do ciclo da vida, buscando, assim, mecanismos de adaptação como a esperança nesta nova fase do desenvolvimento (SOMMERHALDER, 2010).

As dimensões experimentação e realização foram as que apresentaram correlações significativas baixas com a esperança em idosos. Estas duas dimensões são do tipo de orientação pessoal dos valores humanos. A primeira representa as necessidades de sexo, gratificação e do princípio do prazer, enquanto a segunda busca o imediatismo e a realização material e pessoal, caracterizando pessoas focadas no aqui e agora (GOUVEIA et al., 2009). Apesar de terem apresentado correlações positivas significativas com o escore total de esperança, os resultados vão de encontro com o proposto pela teoria funcionalista, em que estas duas dimensões são mais observadas, sobretudo, em adolescentes e jovens adultos (GOUVEIA, 2003), que apresentam uma maior necessidade de experimentar e viver novas sensações, além de ainda estarem em busca da eficiência e sucesso naquilo que se propõem a fazer de sua vida pessoal e profissional, etapas já amplamente vivenciadas pelos idosos. A correlação demonstra que a promoção destes tipos de valores, mesmo que em menor força nesta etapa da vida, também contribui para o desenvolvimento de esperança na terceira idade.

No que se refere ao potencial preditivo dos valores perante a esperança, observou-se que as dimensões existência, suprapessoal, normativa e interativa compuseram o modelo mais estatisticamente significativo para explicar a esperança em idosos. Estes resultados demonstram a ligação dos valores com o fator esperança uma vez que as subfunções existência e suprapessoal formam a espinha dorsal dos valores (valores centrais), estando ligados a necessidades mais básicas como comer e beber, que representam as condições básicas de sobrevivência (existência) ou necessidades ligadas a padrões cognitivos ou de autorrealização (suprapessoal) (GOUVEIA, 2013). O fato de as subfunções normativa e interativa também se apresentarem como preditores significativos da esperança demonstra a importância dos valores sociais para esta etapa da vida. Os valores normativos apresentam-se mais comumente nas pessoas mais velhas uma vez que os valores estão ligados à preservação de cultura e normas (VIONE, 2012), enquanto os valores interativos apresentam-se como 
essenciais para um envelhecimento saudável do sujeito e uma manutenção de sua esperança (STIRLING, 2010).

\section{Considerações finais}

Diante do que foi discutido, considera-se que este estudo possui contribuições relevantes do ponto de vista dos valores humanos, pois buscou avaliar como este construto pode influenciar o sentido da esperança para os idosos. Entretanto, antes de prosseguir com as considerações, ponderam-se as limitações existentes no presente trabalho. A principal, mas não única, é o viés da amostra, que foi considerada por conveniência de forma não probabilística. Esta limitação implica em não poder generalizar os resultados aqui apresentados. Seria importante contar com amostras mais heterogêneas e representativas, sugerindo-se, em estudos futuros, a replicação desta pesquisa com idosos de diferentes populações. Outra limitação foi a falta de referencial teórico ao utilizar os dois construtos conjuntamente, bem como a escassez de estudos sobre valores humanos que utilizem os idosos como amostra uma vez que, comumente, são utilizados adultos (VIONE, 2012).

Outra possível limitação foi o fato do nível instrucional da amostra não ter sido controlado uma vez que $34,6 \%$ da amostra declararam não ter nem mesmo o ensino fundamental completo. O baixo nível instrucional pode ter influenciado nas respostas destes. Além disso, fez-se uso aqui unicamente de medidas de autorrelato, e isso pode ter sido dotado de vieses uma vez que os participantes podem ter respondido dissimuladamente a fim de demonstrar um comportamento socialmente aceitável. Não obstante, mesmo considerando as importantes ponderações acerca das limitações deste estudo, não se pode desconsiderar os resultados encontrados e sua importância.

A população envelhece de forma cada vez mais saudável, fator que não se deve apenas aos avanços da medicina, mas também às mudanças que propiciaram uma melhoria geral no bem-estar das pessoas (BLOOM, 2011). Chegar à velhice saudável demanda mais do que fatores externos, precisando entender que a qualidade de vida depende também do bem-estar subjetivo do indivíduo (SNYDER; RAND; SIGMON, 2002). Por essa razão, ações sistemáticas de diferentes áreas que atuem na melhoria do bem-estar do idoso devem ser projetadas com a finalidade de dar ao idoso o que é de direito, satisfazendo as suas necessidades, respeitando seus limites e promovendo uma esperança de vida ao mesmo. 
Os valores humanos atuam como princípios-guia dos seres humanos, sendo essenciais a partir do momento em que o homem se percebe enquanto indivíduo, adquirindo consciência de suas ações e vendo no outro a possibilidade de existir (GOUVEIA et al., 2011). Eles atuam como um construto central dentro da psicologia (ROKEACH, 1973) e de extrema importância para o entendimento dos fenômenos sociopsicológicos (SCHWARTZ, 1992). Sendo assim, o estudo dos valores humanos associado à esperança apresenta-se como um subsídio que possibilita o planejamento de intervenções mais adequadas com o objetivo de reduzir o impacto da senescência no cotidiano desses indivíduos, fazendo com que o estímulo ao desenvolvimento destes valores (suprapessoal, existência, interativa e normativa) possa atuar fornecendo melhores perspectivas quanto ao futuro aos idosos uma vez que verificou-se a capacidade preditiva destes perante a esperança.

Para estudos futuros, sugere-se a realização de pesquisas longitudinais que averiguem a relação dos valores nas mais variadas fases do desenvolvimento, buscando como este construto pode influenciar na esperança de vida destes. Estudos como este possivelmente poderiam facilitar a visualização da influência que os valores estabelecem no dia a dia das pessoas nas mais variadas faixas etárias.

Destarte, confia-se que o objetivo do presente estudo foi alcançado. Muito embora não haja uma base teórica que explique a relação entre os valores humanos e a esperança, os resultados obtidos foram estatisticamente significativos, indicando uma relação entre os dois construtos. Contudo, fica claro que as mudanças estabelecidas nas relações do idoso com a sociedade ao passar dos anos certamente têm contribuído não só na melhoria dos tratamentos médicos, mas também na mudança de valores, responsáveis por guiar os comportamentos, auxiliando, assim, na promoção da esperança de vida destes idosos.

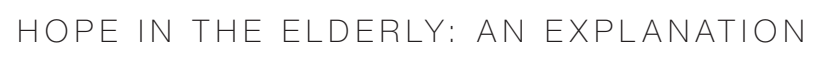

This study aimed to know how hope relates to human values in elderly. The hope is fundamental in the aging process, allowing a new positive perspective about the future. For a better comprehension, it's necessary to consider the human values, which act guiding and directing the human behavior, always present in the society and undergoing changes as the individual matures. 218 elderly participated, with 
a median age of 68 years (SD $=6.65)$, mostly female (61.9\%). We performed Pearson correlations and multiple linear regressions with stepwise method. They answered the Basic Values Questionnaire, the Herth Hope Scale and demographic questions. We performed Pearson correlations between the values subfunctions and the total score of hope, also a multiple regression. Significant correlations were noticed in all the subfunctions: interactive $(r=.43 ; p<.01)$, existence $(r=.39 ; p<.01)$, suprapersonal $(r=.38 ; p<.01)$, normative $(r=.31$; $p<.01)$, promotion $(r=.20 ; p<.01)$ and excitement $(r=.19 ; p<.01)$, respectively. In regression, the interactive, normative, existence and suprapersonal dimensions were significant predictors in the total score of hope, having explained 26\% [F $(4 ; 206)=19,39 ; p<.001]$. The results suggest the importance of the human values in the promotion of a healthier life and with bigger hope in elderly, mainly when refers to central and social values. Concluding, it is considered that the objectives have been achieved, contributing to the literature about quality of life in elderly.

keywords

Hope. Human Values. Elderly.

referências

BLOOM, David E. 7 Billion and Counting. Science, Washington, v. 333, n. 6042, p. 562-569, July 2011.

BRASIL. Lei n 10.741, de $1^{\circ}$ de outubro de 2003. Dispõe sobre o Estatuto do Idoso e dá outras providências. Brasília, DF, out. 2003. Disponível em: <http://www. planalto. gov.br/ccivil_03/leis/2003/110.741.htm>. Acesso em: 4 maio 2014.

Ministério da Saúde. Portaria no 2.528 de 19 de outubro de 2006. Aprova a Política Nacional de Saúde da Pessoa Idosa. Brasília, DF, out. 2006. Disponível em: <http://dtr2001.saude.gov.br/sas/PORTARIAS/Port2006/GM/GM-2528.htm>. Acesso em: 4 maio 2014

Constituição (1988). Constituição [da] Republica Federativa do Brasil. Brasília, DF: Senado Federal. Disponível em: <http://www.planalto.gov.br/ccivil_03/constituicao/ constituicao.htm>. Acesso em: 17 mar. 2014.

CHEAVENS, Jean; GUM, Amber. Gray Power: Hope and Older Adults. In: SNYDER, Charles Richard (Ed.). The Handbook of Hope: Theory, measures, and application. San Diego: Academic Press, 2000. p. 201-222.

DEVESA, Teresa Sofia Moreira Maria. Teste de um modelo preditor de interesses e seguranca em indivíduos do gênero masculino, na adultez, na meia-idade e na velhice. 2005. 50 f. Monografia (Licenciatura em Psicologia) - Instituto Superior de Psicologia Aplicada, Lisboa, 2005. 
FRANKL, Viktor Emil. Logoterapia y análisis existencial: textos de cinco décadas. Barcelona: Herder Editorial, 2007.

GEIGER, Katherine; KWON, Paul. Rumination and depressive symptoms: Evidence for the moderating role of hope. Personality and Individual Differences, Oxford, v. 49 n. 5, p. 391-395, Apr. 2010

GOUVEIA, Valdiney Veloso. A natureza motivacional dos valores humanos: Evidências acerca de uma nova tipologia. Estudos de Psicologia, Natal, v. 8, n. 3, p. 431-443, out. 2003.

- Teoria funcionalista dos valores humanos: fundamentos, aplicações e perspectivas. 1. ed. São Paulo: Casa do Psicólogo, 2013. p. 111-135.

GOUVEIA, Valdiney Veloso et al. Teoria funcionalista dos valores humanos: aplicações para organizações. Revista de Administração Mackenzie, São Paulo, v. 10, n. 3, p. 34-59, maio/jun. 2009.

GOUVEIA, Valdiney Veloso et al. Valores humanos: Contribuições e perspectivas teóricas. In: TORRES, Cláudio Vaz; NEIVA, Elaine Rabelo (Ed.). A psicologia social: Principais temas e vertentes. Porto Alegre: ArtMed, 2011. p. 296-306.

HERTH, Kaye. Abbreviated instrument to measure hope: development and psychometric evaluation. Journal of Advanced Nursing, Oxford, v. 17, n. 10, p. 1251-1259, Oct. 1992

INSTITUTO BRASILEIRO DE GEOGRAFIA E ESTATÍSTICA. Sintese de Indicadores Sociais: uma análise das condições de vida da população brasileira. Rio de Janeiro: IBGE, 2012. Disponível em: < ftp://ftp.ibge.gov.br/Indicadores_Sociais/Sintese_de_Indicadores_Sociais_2012/SIS_2012.pdf >. Acesso em: 4 maio 2014.

INSTITUTO DE ESTUDOS DE SAÚDE SUPLEMENTAR. Envelhecimento populacional e os desafios para o sistema de saúde brasileiro. São Paulo: IESS, 2013. Disponíve em: < http://www.iess.org.br/envelhecimentopop2013.pdf> Acesso em: 4 maio 2014

KAVRADIM, Selma Turan; ÖZER, Zeynep Canli; BOZCUK, Hakan. Hope in people with cancer: a multivariate analysis from turkey. Journal of Advanced Nursing, Oxford, v. 69, n. 5, p. 1183-1196, May 2013.

KÜCHEMANN, Berlindes Astrid. Envelhecimento populacional, cuidado e cidadania: velhos dilemas e novos desafios. Sociedade e Estado, Brasília, DF, v. 7, n. 1, p. 165-180 jan./abr. 2012

MASLOW, Abrahan Harold. Motivation and personality. New York: Harper \& Row, 1954. p. 62-72.

MEDEIROS, Emerson Diógenes de. Teoria funcionalista do valores humanos: testando sua adequação intra e interculturalmente. 2011. 255 f. Tese (Doutorado em Psicologia Social) - Universidade Federal da Paraíba, João Pessoa, 2011

MESTRE, Marina Alexandra Gomes. A esperança e a qualidade de vida na terceira idade. 2011. 187 f. Dissertação (Mestrado em Psicologia) - Instituto Superior Migue Torga, Coimbra, 2011.

NOTHWEHR, Faryle; CLARK, Daniel; PERKINS, Anthony. Hope and the use of behavioural strategies related to diet and physical activity. Journal of Human Nutrition and Dietetics, London, v. 1, n. 1, p. 159-163, July 2013

ORGANIZAÇÃO DAS NAÇÕES UNIDAS. A ONU e as pessoas idosas. Disponível em: <http://nacoesunidas.org/acao/pessoas-idosas/>. Acesso em: 1 ago. 2013

ORLANDI, Fabiana de Sousa et al. Avaliação do nível de esperança de vida de idosos renais crônicos em hemodiálise. Revista da Escola de Enfermagem da USP, São Paulo, v. 46, n. 4, p. 900-905, dez. 2012

ROKEACH, Milton. The nature of human values. New York: Free Press, 1973. 
SARTORE, Alessandra Cristina; GROSSI, Sonia Aurora Alves. Escala de Esperança de Herth: instrumento adaptado e validado para a língua portuguesa. Revista da Escola de Enfermagem da USP, São Paulo, v. 42, n. 2, p. 227-232, jun. 2008.

SCHWARTZ, Shalom House. Universals in the context and structure of values: theoretical advances and empirical tests in 20 countries. In: ZANNA, Mark (Ed.). Advances

in experimental social psychology. v. 25. Orlando, FL: Academic Press, 1992. p. 1-65.

SNYDER, Richard: RAND, Kevin: SIGMON, David. Hope Theory: a member of the Positive Psychology Family. In: SNYDER, Richard; LOPEZ, Shane. (Ed.). Handbook of Positive Psychology. Oxford: Oxford University Press, 2002. p. 257-276.

SOMMERHALDER, Cinara. Sentido de vida na fase adulta e velhice. Psicologia: Reflexão e Crítica, Porto Alegre, v. 23, n. 2, p. 2070-277, jun. 2010.

STIRLING, Elspeth. Valuing older people: Positive Psychology of later life. Chicester: Wiley-Blackwell, 2010. p. 19-23

VAN GESTEL-TIMMERMANS, Hanneke et al. Hope as a determinant of mental health recovery: a psychometric evaluation of the Herth Hope Index-Dutch version. Scandinavian Journal of Caring Sciences, Stockholm, v. 24, Supplement 1, p. 67-74, Nov. 2010

VIONE, Katia Corrêa. As prioridades valorativas mudam com a idade? Testando a hipótese de rigidez e plasticidade. 2012. 151 f. Dissertação (Mestrado em Psicologia) Universidade Federal da Paraíba, João Pessoa, 2012.

Recebido: 09/07/2014 Aceite Final: 26/01/2015 